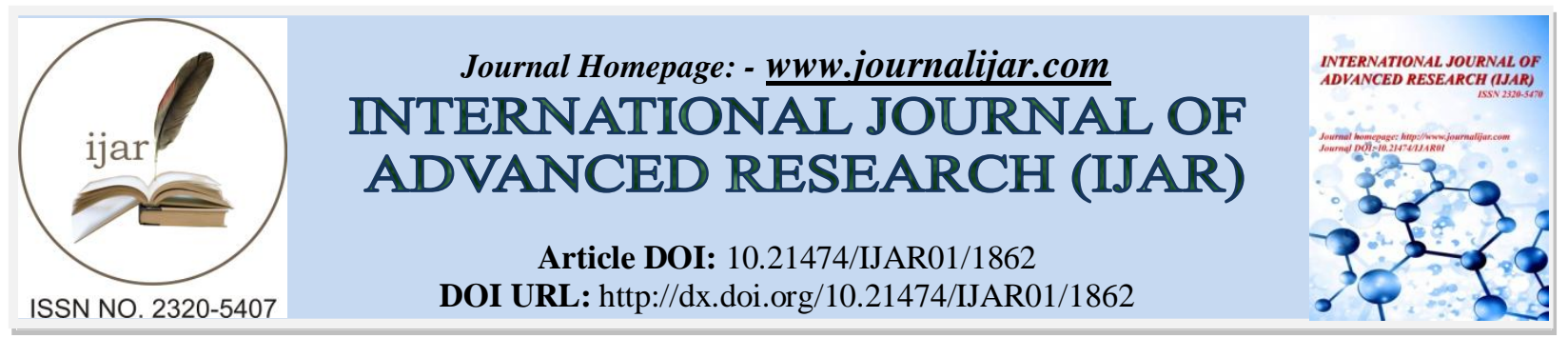

RESEARCH ARTICLE

\title{
TO STUDY THE SAFETY AND EFFICACY OF Nd:YAG HAIR REMOVAL LASER ON PREGNANT AND NON-PREGNANT WOMEN.
}

\author{
Dr. Sajia Hafeez
}

\section{Manuscript Info}

Manuscript History

Received: 12 August 2016

Final Accepted: 22 September 2016

Published: October 2016

Key words:-

postinflammatory hyperpigmentation ,

short term erythema, paradoxical

hirsutism, folliculitis.

\section{Abstract}

Objective:- To study the efficacy and side effects of Nd:YAG laser hair removal in pregnant woman, her conceptus and its comparison to non-pregnant healthy women.

Patients and method:- A retrospective (case control) study was designed in DERMATOLOGY DEPARTMENT,CITY HOSPITAL MULTAN. The study was carried out between June 2013 to June 2016. Total 100 pregnant and 100 non-pregnant healthy women were included in the study. We entered their detailed medical history and clinical examination in proformas. The subjects were divided into two groups. Group A included pregnant and Group B included nonpregnant healthy women. $1064 \mathrm{~nm}$ long pulsed Nd:YAG (Neodymium doped - Yttrium Aluminum Garnet) laser with energy range of 20 to $30 \mathrm{j} / \mathrm{cm}^{2}$ and pulse duration of 3 to 20 millisecond was used in our study. Both groups were given 8 sessions with a gap of eight weeks . Results were assessed by manual counting of hairs and photographs taken.

Results:- GROUP A: Out of 110 subjects, 93 completed the study . 81 $(87.4 \%)$ subjects showed 65 to $75 \%$ reduction of unwanted hairs after eight sessions. 89 (95\%) showed short term erythema. 47 subjects (50.5\%) showed post-inflammatory hyperpigmentation. 19 (20.4\%) showed up blistering and $8.6 \%$ came up with folliculitis. 3 subjects (3.2\%) showed postinflammatory hypopigmentation. 21 subjects $(22.5 \%)$ showed paradoxical hirsutism. 23 (24.7\%) showed perifollicular edema and no subject showed any complication in pregnancy related to HRL and no fetal deformity or anomaly was seen in the neonates after birth. GROUP B: Out of 110 subjects, 103 completed the study. 91 subjects $(88.3 \%)$ showed 65 to $75 \%$ reduction of hairs. $100(97 \%)$ showed short term erythema. 22 $(21.3 \%)$ showed postinflammatory hyperpigmentation. 15 (14.5\%) showed blistering. $6(5.8 \%)$ showed folliculitis. 9 (8.7\%) showed postinflammatory hypopigmentation. $53(51.4 \%)$ showed paradoxical hirsutism. 31 (30\%) showed up perifollicular edema.

Conclusion:- Nd-YAG HRL can be safely used in a pregnant women without any harmful side effects on conceptus. Its equally effective and safe to be used in pregnant and non-pregnant healthy women with minimal side effects. 


\section{Introduction:-}

In our advancing era of applied medical sciences, people including men and women seek the assistance of lasers for the removal of their unwanted body hairs. To our surprise, history of HRLs is also a long one. HRLs were first used by specialists around 1960's . Ruby HRLs were the first one to be used in past. But it had some limitations that hindered to make it a good HRL device. Because it used to injure the surrounding tissue along with the targeted chromophore. Since then different analysis were done and different theories were under consideration in approach for an effective laser treatment without damaging the epidermis and the underlying tissue. The THEORY OF SELECTIVE PHOTOTHERMOLYSIS by Anderson and Perish took the stage. A new sensation began in laser technologies. It was the most effective one uptil now. With the use of THEORY OF SELECTIVE PHOTOTHERMOLYSIS, only the targeted chromophore is damaged sparing the surrounding tissue. Its the most widely used theory uptil now.

Nowadays, different laser devices has been developed. Alexendrite laser, Nd:YAG laser, Ruby laser, Diode laser and IPL are the most frequently used HRL technologies of today. But all of these devices have got some limitations. These technologies are also based on THEORY OF SELECTIVE PHOTOTHERMOLYSIS. So, these devices are basically working better for a dark pigmented hair along with a fair skin complexion. The HRLs available today have the limitations to target and damage eumelanin only; that gives the hair black or dark brown colour. These lasers cannot damage pheomelanin ( chromophore that gives the hair blonde ,red or lighter color). This limits the use of HRLs in skin types having lighter hair color.

Today the use of HRLs in pregnancy is an enigma. Practically, no clinical study has been done so far to ensure the safety and effectiveness of HRLs in a pregnant woman. To our knowledge, no obstetrician or dermatologist throughout the world recommends the use of HRLs in pregnancy. Our study was focused to document the safety and effectiveness of HRLs used in pregnancy as compared to a non pregnant healthy women. And to view the harmful side effects of HRLs on the developing fetus and ongoing pregnancy following serials of HRLs sessions.

In Pakistan, HRLs are increasingly getting popular in all cities and even ladies from villages are also showing interest in HRLs. So, almost every other women is fearing whether HRLs are safe for her or her fetus . SO, to me it was important to rule out the queries associated with HRLs.

\section{Method of study:-}

This was a retrospective study. The study was conducted in CITY HOSPITAL, Multan. We obtained an ethical clearance from the hospital administration. This study was carried out under the strict supervision of Dr Shazia Zubair who is also the head of dermatology department, CITY HOSPITAL. It was conducted from $1^{\text {st }}$ June 2013 to $30^{\text {st }}$ June 2016. The patients were divided into two groups. Group A included 110 pregnant women with a mean age of +30 (22 to 38) years including primigravida and multigravida both. A few of these pregnant ladies were already taking HRL sessions and meanwhile they conceive. And few of them were new candidates for HRLs. All of them were having no previous history of fetal malformation or any significant family history related to fetal deformity or anomaly. Group B contained 110 healthy non-pregnant ladies, with a mean age of +40 years (17 to 63 years). Both groups included patients with Fitzpatrick skin types 2,3,4,5. The patients who were hypertensive or on oral anti hypertensive treatments, diabetics, rosacea, photosensitivity disorders, psoriasis or other skin disorders were excluded from our study. Also females with hormonal disorders, on hormone replacement therapis, polycystic ovarian disease, adrenal insufficiency and pituitary disorders were also excluded from the study. A predesigned proforma was used to enter the detailed past medical history and the clinical examination of the subjects individually.

Long pulsed (1064 nm) Nd:YAG HRL (CANDELA, FDA approved HRL machine) was used. The energy ranges we used were around 20 to $30 \mathrm{j} / \mathrm{cm}^{2}$, with a pulse duration of 3 to 20 millisecond depending on the skin phenotype. We used a contact cooling device, zimmer cryo 5 while using the Nd:YAG HRL machine. It cooled the skin at temperature of -11 to -20 degree centigrade. Our three doctors who were experienced and expert in handeling HRLs did the lasers. All of these three doctors were trained in the HRLs. They used the same technique for all the undergoing subjects. The area ready for HRL treatment was specified and marked. At baseline and after every session of HRL, the hairs from targeted area were manually counted as $2.5 * 2.5 \mathrm{~cm}^{2}$ and photographs were taken ( using 5.7 pixels camera, SAMSUNG NOTE 4 ) and compared by our laser specialist individually. All the subjects 
were asked to come for a follow up visit a week after a HRL session to asses the side effects. The side effects related to HRL were documented in our proforma for each subject individually. The subjects who did not visited us for follow up were taken as drop outs. The assessment of pregnant ladies was also carried out by our expert obstetrician . Anomaly scans of the fetuses were also carried out around 14 to 22 weeks and sonologists were requested for their comments for group A subjects individually. Neonatologists and paediatricians were requested to do the complete medical evauation of the newborns and document any HRLs assosiated side effects.

\section{Results:-}

Out of 110 patients in Group A, 93 completed the study . Out of 93, no anomaly and deformity was seen in fetus . $87.4 \%$ of group A subjects shoed 65 to $75 \%$ reduction of hair removal. And after childbirth no malformation or deformity was found in any neonate. We summarized the results from group A subjects in tabular form. Following are the results collected from group A subjects after having eight sessions with a gap of eight weeks.

Group A:- Results.

\begin{tabular}{|l|l|l|}
\hline Side effects & $\begin{array}{l}\text { Number of subjects } \\
\text { showing s/e }\end{array}$ & Percentage \\
\hline Short-term erythema & 89 & $95 \%$ \\
\hline Post-inflammatory hyperpigmentation & 47 & $50.5 \%$ \\
\hline Blisters formation & 19 & $20.4 \%$ \\
\hline Folliculitis & 8 & $8.6 \%$ \\
\hline Post-inflammatory hypopigmentation & 3 & $3.2 \%$ \\
\hline Paradoxical hirsutism & 21 & $22.5 \%$ \\
\hline Perifollicular edema & 23 & $24.7 \%$ \\
\hline Fetal anomaly or deformity & 0 & $0 \%$ \\
\hline
\end{tabular}

Group B:- Results.

\begin{tabular}{|l|l|l|}
\hline \multicolumn{1}{|c|}{ SIDE EFFECTS } & $\begin{array}{l}\text { Number of subjects showing } \\
\text { S/Es }\end{array}$ & Percentage \\
\hline Short term erythema & 100 & $97 \%$ \\
\hline Post-inflammatory hyperpigmentation & 22 & $21.3 \%$ \\
\hline Blister formation & 15 & $14.5 \%$ \\
\hline Folliculitis & 6 & $5.8 \%$ \\
\hline Postinflammatory hypopigmentation & 9 & $8.7 \%$ \\
\hline Paradoxical hirsutism & 53 & $51.4 \%$ \\
\hline Perifollicular edema & 31 & $30 \%$ \\
\hline
\end{tabular}

In group B,103 subjects completed the study. $88.3 \%$ subjects from this group showed 65 to $75 \%$ reduction of hair after eight sessions . They also showed up in follow ups. After eight sessions given to all subjects with a gap of 2 months, following results were collected.

\section{Discussion:-}

For more than fifty years lasers are in market for the removal of unwanted hairs. There are different varieties of laser devices with different technologies. Amongest them, most commonly used devices are ALEXENDRITE, Nd:YAG ,DIODE, RUBY and IPL HRLs systems. Basically all of them used to destroy the stem cells of hair follicle that are present in the bulge, which supply the follicle with new course of cells cells every day. THEORY of SELECTIVE PHOTOTHERMOLYSIS is the baseline technique of these HRLs devices, helping them to destroy the stem cells. According to this theory, the eumelanin chromophore is targeted. Eumelanin is responsible for the black or dark brown color of the hairs. Eumelanin is destroyed only in anagen phase of hair cycle. It is because follicular melanogenesis occurs only in anagen phase of hair cycle. It is totally switched off in catagen phase and is completely absent in telogen phase. That's why eumelanin is targeted only in anagen phase ${ }^{1,2}$.

To our knowledge and all available record up till now, no any detailed research is done to define the safety of HRLs used in pregnancy. The obstetricians, dermatologists and even laser specialists throughout the world do not recommend HRLs to be used in pregnancy. For very little knowledge is available in this regard. Our study was 
based on this issue, to resolve the mystery whether the HRLs are safe to be used in pregnancy and to document the associated side effects on the developing fetus.

In pregnancy, the anagen phase of hair cycle is lengthened. Therefore, more hairs on the body are in anagen phase in pregnancy as compared to a non-pregnant body. Due to hormonal fluctuations in pregnancy, the number of hairs is also increased in pregnany. In our research study, all of the under observation pregnant ladies of group A went through eight sessions of HRLs .Long pulsed Nd:YAG, CANDELA ( $1064 \mathrm{~nm}$ ) was used. The energy was 20 to 30 $\mathrm{j} / \mathrm{cm}^{2}$, pulse duration was kept around 20 to 3 milliseconds. The pulse and fluence were adujsted according to the skin phenotype of the subjects and thickness of hair. And a contact cooling device, Zimmer cryo 5 was used to cool the skin during the procedure. At baseline, and after each session the hairs on the treated area were counted manually $\left(2.5 * 2.5 \mathrm{~cm}^{2}\right)^{3}$, photographs were also taken under the same light and using the same camera. Results were compared and assessed by our team of expert doctors who were also experienced at doing HRLs. All of our experts used the same technique for laser. It was noticed that after first session the reduction of hairs was 30 to 40 percent. After each successive session the reduction was almost 35 to 45 percent and increased hair reduction was notices with each upcoming session. After a serial of eight sessions, $87.4 \%$ of the pregnant ladies showed the result of 65 to $75 \%$ reduction of unwanted hair. As more hairs were in anagen phase, thus more hairs were targeted. The underwent subject were quite satisfied with loss of more hairs as compared to their HRLs in non-pregnant state. And as compared to group B patients, their manual hair counting showed more reduction .

97\% of group A patients showed erythema after the laser procedure. It was short term and intensity ranged from mild to moderate. In some subjests erythema was accompanied by edema. That was also mild to moderate. It tended to resolve within two hours to fourty eight hours. We also applied topical mometasone on the effective area to reduce the erythema and edema ${ }^{4,5,6}$.

$50 \%$ of the subjects in group A showed post-inflammatory hyperpigmentation . In few of the patients, it was associated with scarring of the epidermis. It remained quite worrisome for our subjects too. It did not resolve by application of local ointments. $20.4 \%$ of the patients also showed up with blisters formation with scarring. It resolved spontaneously over a span of one week after the laser session with application of any topical ointment. $8.6 \%$ of the patients presented with folliculitis. Oral and topical antibiotics solved the problem.

Paradoxical hirsutism remained the main issue of our subjects. The low levels of laser energy transmitted from the target area stimulated the neighboring hairs. Although laser sessions covered it too, but we had to increase the areas which also increased the cost. Perifollicular edema was also self limiting. The major point to concern was the developing fetus and ongoing pregnancy. Therefore, no complication was seen in pregnancy of group A subjects. All of the under treatment group A subjects delivered healthy babies. Even these patients continued their laser sessions during lactation without any remarkable complication.

Group B patients also went through eight laser session using the same technique. The gap between the sessions was also eight weeks. They also showed up almost similar problems comparable to Group A subjects. But postinflammatory hyperpigmentation was less remarkable. $88.3 \%$ of the patients showed 65 to $75 \%$ of hair reduction after serials of eight sessions. The side effects like short term erythema, blistering, vesiculation, transient pigmentory alterations, fibrosis on scarring and perifollicular edema were noticed ${ }^{7,8}$. All of these side effects were self limiting $.53 \%$ of the patients faced paradoxical hirsustism. It led to patient's disappointment and distrust on HRLs ${ }^{9}$.

In both groups, 22 different anatomical body areas were treated with Nd:YAG HRL . Facial and body hairs showed almost the same result after 8 sessions. But pubic and axillary hairs showed 65 to $75 \%$ reduction even after five HRL sessions. Thus, thick and coarse terminal hairs were better to treat with HRLs. It was also seen that larger the head of Nd:YAG the more effective was the result. The laser beams penetrate the skin as deeper as the size of the head. No deep tissue penetration was recorded.

Thus, the enigma; whether Nd:YAG HRL used in a pregnant woman is safe or not seems to be solved. It does not penetrate the deeper tissue $1^{10,11}$. The need of the day is to find a better HRL technique with lesser pain during treatment. We are also looking forward to more advance technologies for the reduction of unwanted grey or blonde hairs too ${ }^{13,14}$. 


\section{References:-}

1. Goldberg D.J, Litter CM,Wheeland RG. Topical suspension assisted Q-switched Nd:YAG laser hair removal. Dermatol Surg. 1997 Sep. 23(9):741-5

2. Nanni CA,Alster TS. Optimizing treatment parameters for hair removal using a topical carbon based solution and 1064 nm Q-switched neodymium:YAG laser energy. Arch Dermatol 1997 Dec. 133 (12):1546-9.

3. Alster TS, Bryan H, Williams CM. Long pulsed Nd:YAG assisted hair removal in pigmented skin : a clinical and histological evaluation . Arch Dermatol. 2001 Jul. 137(7):885-9.

4. Raff K, Landthaler M, Hohenleutner U. Optimizing treatment parameters for hair removal using long pulsed Nd:YAG laser. Lasers Med. Su. 2004. 18(4):219-22.

5. Tanzi EL, Alster TS. Long pulsed $1064 \mathrm{~nm} \mathrm{Nd:YAG} \mathrm{laser} \mathrm{assisted} \mathrm{hair} \mathrm{removal} \mathrm{in} \mathrm{all} \mathrm{skin} \mathrm{types.Dermatol}$ Surg. 2004. Jan. 30 (1):13-7.

6. Khoury JG, Saluja R, Goldman MP. Comparative evaluation of long pulsed Alexendrite and long pulsed Nd:YAG laser systems used individually and in combination for axillary hair removal. Dermatol Surg. 2008 May. 34(5):665-70; discussion 670-1.

7. Vachiramon V, Brown T, Mc Michel A J . Patients satisfaction and complications following laser hair removal in ethnic skin. J Drugs Dermatol 2012 Feb. 11(2):192-5.

8. Aydin F, Pancar G S, Senturk N, Bek Y, Yuksel E P, Canturk T, et al. Axillary hair removal with 1064-nm Nd:YAG laser increases sweat production. Clin Exp Dermatol 2010 Aug. 35(6):588-92.

9. Radmanesh M. Azar - Beig M, Abtahian A, Naderi A H. Burning, paradoxicall hypertrichosis, leukotricia and folliculitis are four major complications of intense pulse light hair removal therapy. J Dermatology Treat. 2008. 19(6):360-3.

10. Casy A S, Goldberg D. Guidelines for hair removal laser. J Cosmet Laser Ther. 2008 Mar 10(1):24-33

11. Tierney E P, Goldberg D J. Laser hair removal pearls. J Cosmet Laser Ther. 2008 March. 10(1):17-23.

12. Sand M, Bechara F G, Sand D, Altmeyer P, Hoffmann K. A randomized, controlled, double blind study evaluating melanin encapsulated Liposomes as a chromophore for laser hair removal of blonde, white and grey hair. Ann Plast Surg. 2007 May. 58(5):551-4.

13. Gar SD, Graber EM. Laser hair removal, a Review. Dermatol Surg. 2013 Jun.39(6):823-38.

14. Dierickx CC, Grossman MC, Farinelli WA, Anderson RR. Permanent hair-removal by normal-mode Ruby laser. Arch Dermatol. 1998 Jul.134(7):837-42. 